\title{
Photoinduced entropy of InGaN/GaN p-i-n double-heterostructure nanowires
}

\author{
Nasir Alfaraj, ${ }^{1}$ Somak Mitra, ${ }^{2}$ Feng Wu, ${ }^{1}$ Idris A. Ajia, ${ }^{2}$ Bilal Janjua, ${ }^{3}$ Aditya Prabaswara, ${ }^{3}$ \\ Renad A. Aljefri, ${ }^{1}$ Haiding Sun, ${ }^{1}$ Tien Khee $\mathrm{Ng},{ }^{3}$ Boon S. Ooi, ${ }^{3}$ Iman S. Roqan, ${ }^{2, a)}$ \\ and Xiaohang $\mathrm{Li}^{1, \mathrm{~b})}$ \\ ${ }^{1}$ Advanced Semiconductor Laboratory, King Abdullah University of Science and Technology (KAUST), \\ Thuwal 23955-6900, Kingdom of Saudi Arabia \\ ${ }^{2}$ Semiconductor and Material Spectroscopy Laboratory, King Abdullah University of Science and Technology \\ (KAUST), Thuwal 23955-6900, Kingdom of Saudi Arabia \\ ${ }^{3}$ Photonics Laboratory, King Abdullah University of Science and Technology (KAUST), Thuwal 23955-6900, \\ Kingdom of Saudi Arabia
}

(Received 23 January 2017; accepted 6 April 2017; published online 19 April 2017)

\begin{abstract}
The photoinduced entropy of $\mathrm{InGaN} / \mathrm{GaN}$ p-i-n nanowires was investigated using temperaturedependent $(6-290 \mathrm{~K})$ photoluminescence. We also analyzed the photocarrier dynamics in the InGaN active regions using time-resolved photoluminescence. An increasing trend in the amount of generated photoinduced entropy of the system above $250 \mathrm{~K}$ was observed, while we observed an oscillatory trend in the generated entropy of the system below $250 \mathrm{~K}$ that stabilizes between 200 and $250 \mathrm{~K}$. Strong exciton localization in indium-rich clusters, carrier trapping by surface defect states, and thermodynamic entropy effects were examined and related to the photocarrier dynamics. We conjecture that the amount of generated photoinduced entropy of the system increases as more non-radiative channels become activated and more shallowly localized carriers settle into deeply localized states; thereby, additional degrees of uncertainty related to the energy of states involved in thermionic transitions are attained. Published by AIP Publishing.
\end{abstract}

[http://dx.doi.org/10.1063/1.4981252]

In recent years, it has become clear that integrating various forms of inorganic materials, based on group-III-nitride compound semiconductors and silicon, into one electronic system shows promise in reshaping the future of the electronics industry. ${ }^{1-11}$ Group-III-nitride nanowires are capable of realizing high-performance semiconductor optoelectronic devices with complex heterostructures ${ }^{12,13}$ because the nanowire sidewalls permit strain reduction, which allows for the combination of extremely lattice-mismatched materials without producing dislocations. ${ }^{14}$ Together with the advantages of the sharp peaks of density of states at the lowest quantized sub-band energy levels, improved exciton binding energy, and increased wavefunction overlap of the electron-hole pairs, GaN-based nanowires have the potential for realizing integrated optoelectronic systems because of their superior quantum efficiencies. ${ }^{15}$ GaN-based nanowires are also expected to pave the way for new photodetector device architectures, improve photosensitivity, and reduce polarization fields. ${ }^{16-18}$ Low $\mathrm{NiO} / p^{+}-\mathrm{GaN}$ contact resistance can be realized thanks to hole tunneling through the potential barrier at the $\mathrm{NiO} / p^{+}-\mathrm{GaN}$ interface, which significantly enhances the energy-saving performance of GaN-based p-i-n power devices. ${ }^{19}$ High-level injection nitride-based $p-i-n$ junction nanowires are suitable for attenuators, high-frequency switches, as well as photodetector applications. ${ }^{20}$ However, non-radiative surface recombination and the filling of innershell vacancies affect the performance of devices based on such nanowires because the quantum efficiency of nanowire optoelectronic devices can be limited by the non-radiative Shockley-Read-Hall (SRH) ${ }^{21}$ and Auger $^{22}$ recombination

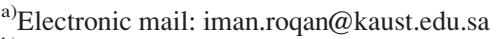

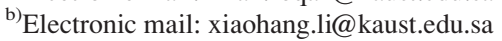

processes. Because of higher surface-to-volume ratios and the subsequent formation of surface trap states, the nonradiative photocarrier recombination lifetimes in nanostructured materials are typically shorter than those of their bulk counterpart. In the specific case of group-III-nitride semiconductors, it is generally accepted that the photocarrier relaxation dynamics consists of an initial decay process in the subpicosecond range, which is followed by a slow decay described by the ultrafast carrier thermalization dynamics, the carrier trapping by surface states, and the slower carrier cooling effects, ${ }^{23}$ respectively. This process provides radiant refrigeration, suggesting that they are solid-state radiative heat pumps $^{24}$ as illustrated by David et al. for the case of group-IIInitride light-emitting diodes (LEDs). ${ }^{25}$ Hence, a more in-depth understanding of the opto-electrothermal properties of groupIII-nitride nanowires through a new entropic perspective is necessary to further understand their applicability and operational and thermal stability ${ }^{26}$ in multifunctional applications. ${ }^{27}$

In this study, we employed temperature-dependent photoluminescence (PL) to investigate the photoinduced entropy of InGaN/GaN p-i-n double-heterostructure nanowire photodiodes. We defined the photoinduced entropy as a thermodynamic quantity that represents the unavailability of a system's energy for conversion into work due to luminescence refrigeration. ${ }^{28}$ We also studied the ultrafast dynamics of photocarriers using wavelength-integrated time-resolved photoluminescence (TRPL) down to the subnanosecond regime. We chose to study the thermodynamic behavior and photocarrier dynamics in GaN-based p-i-n nanowires because a thorough understanding of the factors that govern the energy transfer processes, and the transport of free carriers through them, is crucial to realize effective designs of reliable and efficient $\mathrm{p}-\mathrm{i}-\mathrm{n}$ high-power electronics and photodetecting 
(a)
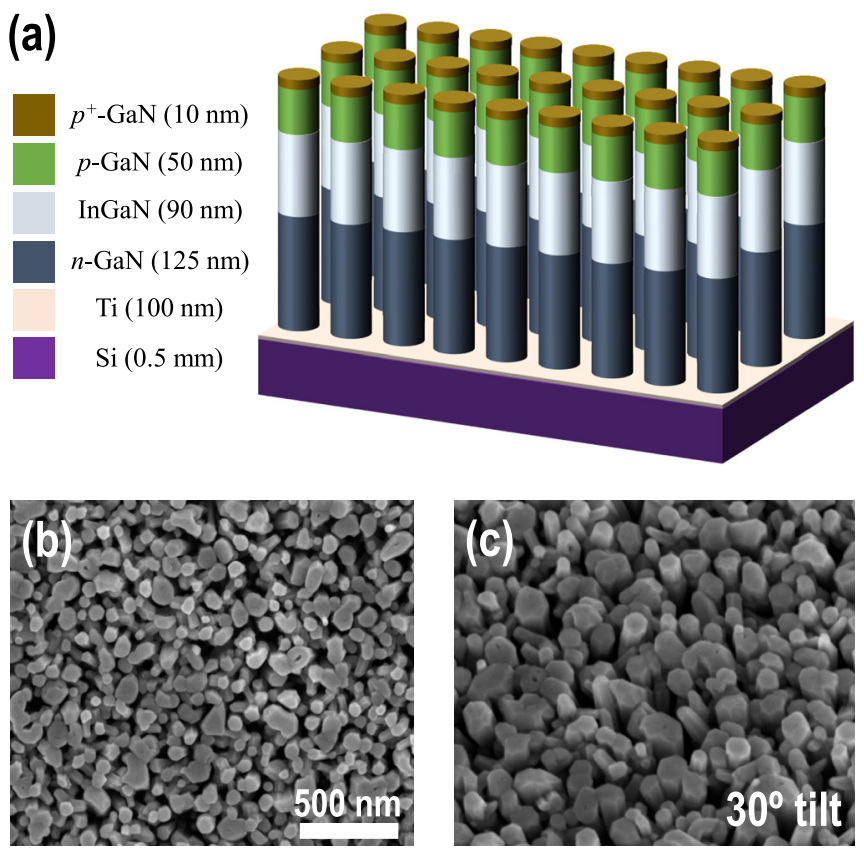

FIG. 1. (a) Schematic and layer structure of the InGaN/GaN p-i-n nanowires, (b) plan-view, and (c) elevation-view SEM images of the nanowires.

devices. Fig. 1(a) shows the schematic layer structure of our nanowires, with the $c$-axis along the growth direction normal to the surface. The p-i-n nanowires were grown on a titaniumcoated silicon substrate using plasma-assisted molecular beam epitaxy (PA-MBE). ${ }^{29}$ The nanowires comprised $125 \mathrm{~nm}$ silicon-doped $n$-GaN (dopant carrier concentration $\left(N_{\mathrm{d}}\right) \approx$ $\left.5 \times 10^{18} \mathrm{~cm}^{-3}\right), 90 \mathrm{~nm}$ unintentionally doped InGaN, $50 \mathrm{~nm}$ magnesium-doped $p$-GaN $\left(N_{\mathrm{a}} \approx 10^{18} \mathrm{~cm}^{-3}\right)$, and $10 \mathrm{~nm}$ magnesium-doped $p^{+}-\mathrm{GaN} \quad\left(N_{\mathrm{a}} \approx 5 \times 10^{18} \mathrm{~cm}^{-3}\right)$ layers grown sequentially. The morphology of the nanowires was characterized using an FEI Magellan field emission gun (FEG) field-emission scanning electron microscope (FE-SEM).

PL and TRPL measurements were performed with a mode-locked Ti:sapphire laser (Coherent Mira 900) having a laser power output of $1.90 \mathrm{~W}$ at $800 \mathrm{~nm}$, and the measurements were used to study the carrier lifetimes in the active InGaN layers. A third harmonic generator (APE-SHG /THG) was used to excite the sample by an output wavelength $\left(\lambda_{\mathrm{ex}}\right)$ of $266 \mathrm{~nm}$ (pulse width [ $\left.\tau_{\text {on }}\right] 150 \mathrm{fs}$, pulse repetition rate $\left[f_{\text {rep }}\right]$ $76 \mathrm{MHz}, 200$ integrations collected with a time-delay integration [TDI] of $100 \mathrm{~ms}$ ) over a temperature range from $6 \mathrm{~K}$ to 290 K. Emission of the sample was detected by a monochromator attached to a UV-sensitive Hamamatsu C6860 streak camera with a temporal resolution of $2 \mathrm{ps}$. The samples were mounted in a variable temperature closed helium cryostat (Janis). Fig. 1(b) depicts a plan-view SEM image, where the nanowire density $\left(\rho_{\mathrm{NW}}\right)$ is about $3.37 \times 10^{10} \mathrm{~cm}^{-2}$, with a mean cross-sectional size $\left(2 r_{\mathrm{NW}}\right)$ of $55 \mathrm{~nm}$, while Fig. 1(c) depicts an elevation-view SEM image of the nanowires.

Fig. 2 shows the simulated band diagram of the nanowires, assuming a band-offset ratio of around 70:30 (a commonly assumed value for group-III-nitride semiconductors), ${ }^{30,31}$ which enables the visualization of surface defects and provides insight into the local potential fluctuations in the structure.

In Figs. 3(a) and 3(b), we show typical PL and TRPL decay curves, respectively, for an ensemble of InGaN active

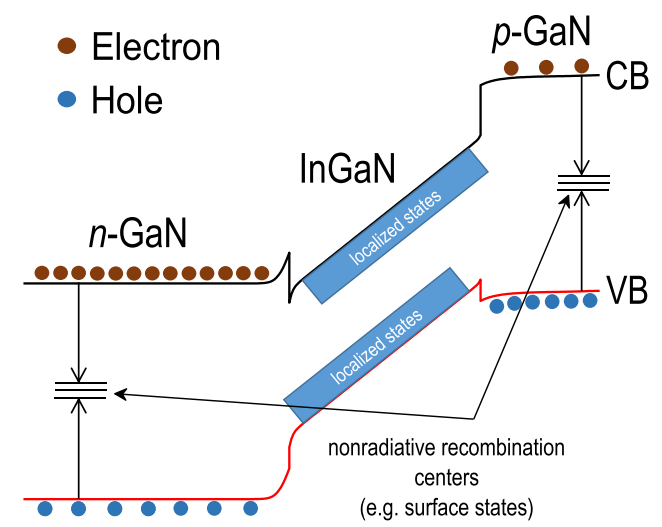

FIG. 2. Schematic illustration of the energy band diagram of the InGaN/ GaN p-i-n nanowires in equilibrium, taking into account spontaneous and piezoelectric polarization fields.

layers at $6 \mathrm{~K}$ and $290 \mathrm{~K}$. The TRPL decay curves were analyzed using a two-term exponential decay model. We chose to fit our TRPL decay curves using a two-term exponential decay model because it better depicts the multiple recombination characteristics exemplified by our TRPL curves. ${ }^{32}$ Put differently, the two-term exponential model best fits the ultrafast carrier thermalization dynamics (the initial fast decay process) and the slower carrier cooling effects (slow decay processes) that are characteristic of our measured
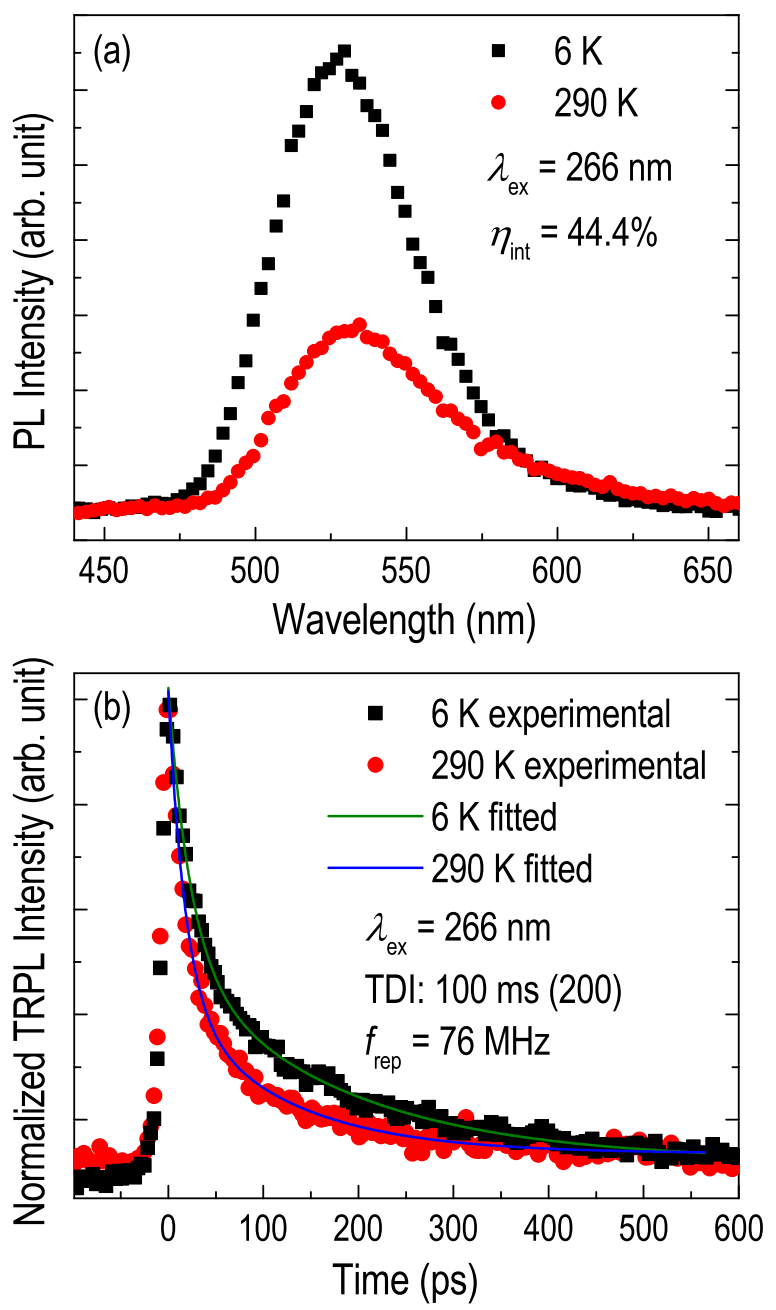

FIG. 3. Typical InGaN-related (a) PL and (b) TRPL spectra for the InGaN/ $\mathrm{GaN}$ p-i-n nanowires at $6 \mathrm{~K}$ and $290 \mathrm{~K}$. 
InGaN active layers, thus enabling the analysis of the fast and slow components separately. ${ }^{33}$

Because we are measuring the InGaN-related PL and TRPL using an ultrashort-pulse laser, it is imperative to investigate the Auger-induced effects on the internal quantum efficiency $\left(\eta_{\text {int }}\right)$ in the measured InGaN layers. Temperaturevariable $\eta_{\text {int }}$ values $\left(\eta_{\text {int }}(T)\right)$ are crucial parameters that we will use to calculate the carrier recombination lifetimes. The photogenerated carrier density was estimated using the following formula ${ }^{34}$

$$
\rho_{\mathrm{ph}}=\frac{\alpha(1-R) P_{\mathrm{ex}}}{E_{\mathrm{ph}} f_{\mathrm{rep}} A_{\mathrm{avg}}},
$$

where $\alpha$ is the InGaN absorption coefficient, $R$ is the reflectivity at the GaN-air interface, which is about $20 \%$ at $\lambda_{\mathrm{ex}}=266$ $\mathrm{nm},{ }^{35} P_{\mathrm{ex}}$ is the laser average excitation power $(3.6 \mathrm{~mW}), E_{\mathrm{ph}}$ is the incident photon energy $\left(h c / \lambda_{\mathrm{ex}}\right)$, and $A_{\text {avg }}$ is the laser beam area $\left(\pi r^{2}, r \approx 20 \mu \mathrm{m}\right)$. The indium composition in the active InGaN layers was estimated using Vegard's law and found to be approximately $32 \%$. Therefore, we estimated $\alpha$ at $\lambda_{\mathrm{ex}}=266 \mathrm{~nm}$ to be roughly $1.74 \times 10^{5} \mathrm{~cm}^{-1} ;{ }^{34}$ hence, $\rho_{\mathrm{ph}}$ was estimated to be around $6.95 \times 10^{17} \mathrm{~cm}^{-3}$, which is well below typical Auger-inducing carrier densities in $\mathrm{InGaN}^{36}$ and falls around the optimal carrier density level $\left(N_{\text {carrier }} \approx 10^{18} \mathrm{~cm}^{-3}\right)$ for estimating $\eta_{\text {int }} \cdot{ }^{37}$ Furthermore, the peak pulse power intensity $\left(I_{\text {pulse }}\right)$ was estimated using ${ }^{38}$

$$
I_{\text {pulse }}=\frac{P_{\mathrm{ex}}}{\tau_{\mathrm{on}} f_{\mathrm{rep}} A_{\mathrm{avg}}} .
$$

This yields $I_{\text {pulse }}=25.13 \mathrm{MW} / \mathrm{cm}^{2}$, which is well below typical Auger-inducing power densities of more than $100 \mathrm{MW} /$ $\mathrm{cm}^{2} .{ }^{39}$ We used the pulsed-excitation PL measurements to evaluate $\eta_{\text {int }}$ of the measured InGaN active layers at room temperature, which was estimated to be $44.4 \%$ using the ratio of integrated PL intensity at $290 \mathrm{~K}$ to the PL intensity at $6 \mathrm{~K}$, as shown in Fig. 3(a)-assuming that the non-radiative recombination centers are thermally inert at $6 \mathrm{~K}^{40,41}$

Fig. 4(a) shows the temperature-dependent variation in the peak energy of the photon emission $\left(E_{\mathrm{PL}, \text { peak }}\right)$ from the active InGaN layers, which was fitted using the band-tail model, ${ }^{42,43}$ where $\gamma$ and $\beta$ are the Varshni coefficient and Debye temperature, respectively. The parameter $\sigma$ indicates the degree of carrier localization in the InGaN active regions. It has been argued that the number of emitted photons from an active region is limited by the entropy of the system, which must remain positive. This fact is indicated by the second law of thermodynamics-emitted photons transport entropy produced by the absorption of heat from the active region, which acts as a Carnot heat engine. ${ }^{25,44,45}$ The Sshaped behavior depicted by the $E_{\mathrm{PL} \text {, peak }}$ curve in Fig. 4(a) suggests the presence of localized states induced by potential fluctuations in indium-rich clusters within the active InGaN layers. ${ }^{46-48}$ These states are introduced by the enhanced formation of In-N chains in the immediate vicinity of the threading dislocations, hence limiting non-radiative recombination of carriers at the dislocation cores. ${ }^{49,50}$ As observed in Fig. 4(a), $E_{\mathrm{PL} \text {, peak }}$ redshifts $21 \mathrm{meV}$ as the temperature increases from $6 \mathrm{~K}$ to $150 \mathrm{~K}$ compared to the expected
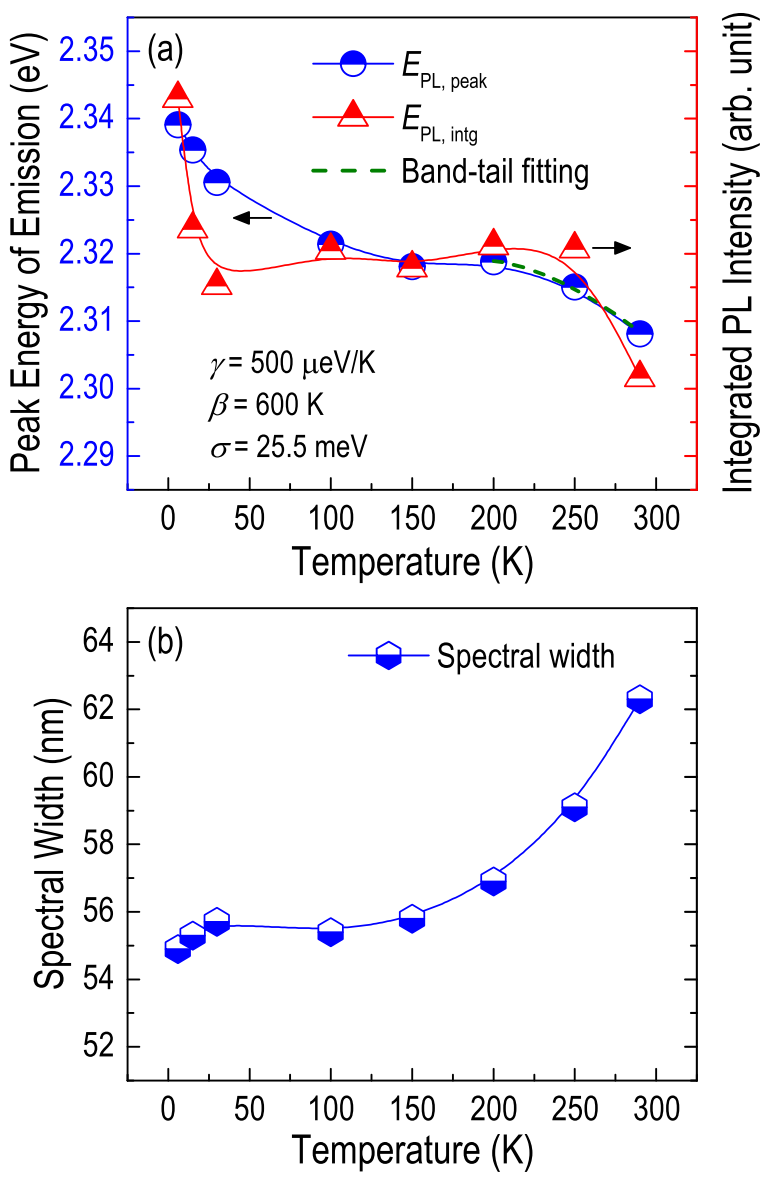

FIG. 4. (a) The InGaN-related $E_{\mathrm{PL}, \text { peak }}$ and $E_{\mathrm{PL} \text {, intg }}$ curves and (b) the spectral width of PL spectra as a function of temperature.

bandgap shrinkage value of approximately $11 \mathrm{meV}$, which corresponds to an effective redshift of $9 \mathrm{meV}$ and a slope of $-62.5 \mu \mathrm{eV} / \mathrm{K}$.

Recall that the thermodynamic entropy $S$ of a system with a total energy $E$ at temperature $T$ satisfies

$$
\frac{\partial S}{\partial E}=\frac{1}{T} .
$$

Let $E_{1}<\cdots<E_{8}$ denote the sorted total energies, and let $T_{\mathrm{j}}$ be the system temperatures (i.e., $6,15,30,100,150,200$, 250 , and $290 \mathrm{~K}$ ) corresponding to each $E_{\mathrm{j}}$. We may approximate the above equation as

$$
\frac{S\left(E_{\mathrm{j}+1}\right)-S\left(E_{\mathrm{j}}\right)}{\left(E_{\mathrm{j}+1}-E_{\mathrm{j}}\right)} \approx \frac{2}{T_{\mathrm{j}+1}+T_{\mathrm{j}}} .
$$

Because we are investigating photoinduced entropy generation, we assume that the net energy exchange is due to photoexcitation and photoluminescence. In our case, the total energy exchanged during each photoexcitation process can be calculated as $E_{\mathrm{PL}, \text { intg }}-N_{\mathrm{ph}} E_{\mathrm{ph}}$, where $N_{\mathrm{ph}}$ is the total

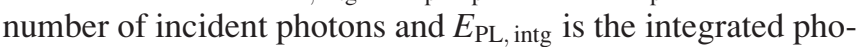
ton energy emission. Therefore, because $N_{\mathrm{ph}} E_{\mathrm{ph}}$ is constant with temperature, we may use

$$
E_{\mathrm{j}+1}-E_{\mathrm{j}}=E_{\mathrm{j}+1}^{\mathrm{PL}, \text { intg }}-E_{\mathrm{j}}^{\mathrm{PL}, \text { intg }}
$$

in Equation (4) to obtain the change in entropy due to photoexcitation as the solution to the following recurrence relation 


$$
S\left(E_{\mathrm{j}+1}\right) \approx S\left(E_{\mathrm{j}}\right)+2 \frac{\left(E_{\mathrm{j}+1}^{\mathrm{PL}, \text { intg }}-E_{\mathrm{j}}^{\mathrm{PL}, \text { intg }}\right)}{T_{\mathrm{j}+1}+T_{\mathrm{j}}} .
$$

We used Equation (6) to qualitatively study the relative entropy generation at $T_{\mathrm{j}+1}$, with respect to an initial arbitrary value at $T_{1}$ (i.e., $T=6 \mathrm{~K}$ ). We also calculated the total carrier recombination lifetimes $\left(\tau_{\text {tot }}\right)$ using the PL and TRPL measurements. After determining $\tau_{\text {tot }}$ and relative $\eta_{\text {int }}$ values at each temperature with respect to $290 \mathrm{~K}$, we calculated the radiative $\left(\tau_{\mathrm{r}}\right)$ and non-radiative $\left(\tau_{\mathrm{nr}}\right)$ carrier lifetimes using $\tau_{\mathrm{r}}(T)=\tau_{\text {tot }}(T) / \eta_{\text {int }}(T)$ and $\tau_{\text {nr }}(T)=\tau_{\text {tot }}(T) /\left(1-\eta_{\text {int }}(T)\right) .{ }^{51}$

Fig. 5(a) depicts the calculated $\tau_{\mathrm{r}}$ and $\tau_{\mathrm{nr}}$, while $\tau_{\text {tot }}$ and the entropy generation are plotted in Fig. 5(b). We observed that $\tau_{\text {tot }}$ values were in the range of $135-178$ ps over the temperature range of $6 \mathrm{~K}$ to $290 \mathrm{~K}$. The absence of quantum confinement effects in our InGaN active layers resulted in less efficient radiative recombination of photoexcited carriers in the presence of competition from carrier localization states because carrier localization increases the radiative recombination efficiency. ${ }^{52,53}$ As depicted in Fig. 5(b), we observed an oscillatory behavior in the generated entropy of the system below $250 \mathrm{~K}$ that stabilizes between $200 \mathrm{~K}$ and $250 \mathrm{~K}$, which can be attributed to the random distribution among deeply and shallowly localized states. As the temperature increased, more shallowly localized carriers settled into broadly localized states through hopping, ${ }^{54}$ which is a form of the band filling effect, contributing to broader spectral widths of the PL spectra as observed in Fig. 4(b). Once the temperature exceeded $250 \mathrm{~K}$, more non-radiative channels
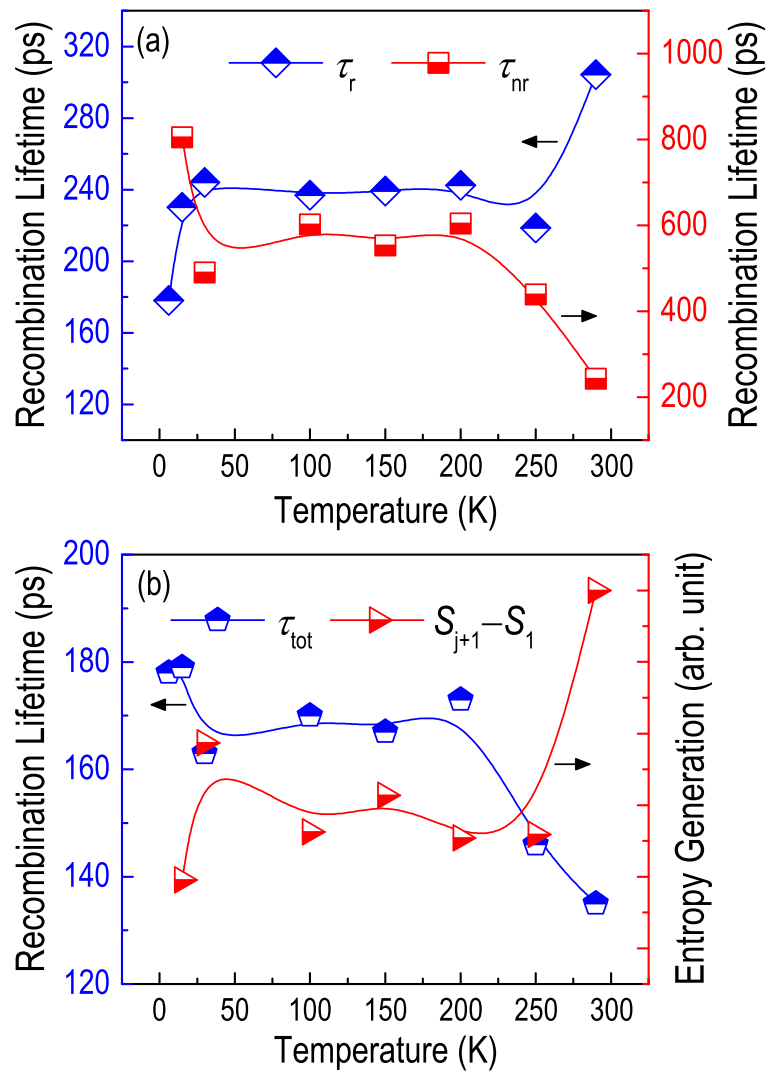

FIG. 5. (a) The extracted $\tau_{\mathrm{r}}$ and $\tau_{\mathrm{nr}}$ of the InGaN active layers and (b) the calculated $\tau_{\text {tot }}$ and evolution of the amount of entropy generation with temperature. became activated as much of the shallowly localized carriers settled into the broadly distributed deeply localized states, and the amount of generated photoinduced entropy of the system increased dramatically, contributing to the uncertainty related to the energy of states involved in thermionic transitions. The decrease in $\tau_{\text {tot }}$ with increasing temperature reflects the fact that $\tau_{\mathrm{nr}}$ decreases, which we attribute to surface defects. ${ }^{55,56} \mathrm{We}$ attribute the increase in $\tau_{\text {tot }}$ at low temperatures to the thermal annihilation of non-radiative recombination centers. ${ }^{51} \mathrm{We}$ hypothesize that non-radiative recombination due to the activation of non-radiative recombination channels contributes to the overall increasing trend in the entropy above $250 \mathrm{~K}$. At this point $(T>250 \mathrm{~K})$, we can confidently state that the non-radiative recombination processes have become dominant, and the lifetimes dropped gradually as the photogenerated carriers are less affected by the fast changes in carrier lifetimes, ${ }^{46,51}$ which cause even higher degrees of uncertainty related to the energy of states involved in thermionic transitions, hence the rise in photoinduced entropy, which can be observed in Fig. 5(b).

In the ideal Carnot cycle (where processes are reversible), there is no generation of entropy, and entropy is therefore conserved. However, in the presence of non-radiative recombination centers in our nanowires due to surface states and other defects, additional entropy is generated because of the irreversible nature of non-radiative processes. ${ }^{25}$ Moreover, for $T>200 \mathrm{~K}$, we observe in Fig. 5(a) that the rate of dissociation between radiative and non-radiative recombination processes increased as other non-radiative processes started to kick in, at which point the total carrier lifetime began to decrease with increasing temperatures. In addition to carrier hopping into the broadly distributed localized states, this decrease in carrier lifetimes caused the carriers to recombine before reaching the lower tails of energy states, which as a consequence of Heisenberg's uncertainty principle explains the broadening of the $E_{\mathrm{PL} \text {, peak }}$ curve as observed in the spectral width plot in Fig. $4(\mathrm{~b}){ }^{46}$

In summary, we investigated the photoinduced entropy of InGaN/GaN p-i-n nanowires and analyzed the InGaNrelated photocarrier dynamics. In addition to defect states, we conjecture that the thermal energizing of shallowly localized states, where the lifetimes are affected by thermal activation, results in carrier relaxation into broadly distributed deeply localized states via hopping, thus increasing the amount of generated randomness in the InGaN layers as the temperature rises. These effects are manifested in the oscillatory behavior $(T<250 \mathrm{~K})$ and increasing trend $(T>250 \mathrm{~K})$ in entropy generation.

This publication is based upon work supported by the King Abdullah University of Science and Technology (KAUST) baseline funding, BAS/1/1664-01-01 and BAS/1/ 1647-01-05. B.J., A.P., T.K.N., and B.S.O. acknowledge the financial support from King Abdulaziz City for Science and Technology (KACST), Grant No. KACST TIC R2-FP-008, and KAUST baseline funding, BAS/1/1614-01-01. The authors would like to thank Professor Enzo Di Fabrizio, Mr. Meshal Alawein, and Mr. Wael Alghamdi of KAUST for their helpful suggestions. 
${ }^{1}$ T. Detchprohm, Y.-S. Liu, K. Mehta, S. Wang, H. Xie, T.-T. Kao, S.-C. Shen, P. D. Yoder, F. A. Ponce, and R. D. Dupuis, Appl. Phys. Lett. 110, 011105 (2017).

${ }^{2}$ A. W. Bruch, K. Xiong, H. Jung, X. Guo, C. Zhang, J. Han, and H. X. Tang, Appl. Phys. Lett. 110, 021111 (2017).

${ }^{3}$ J. Sellés, V. Crepel, I. Roland, M. El Kurdi, X. Checoury, P. Boucaud, M. Mexis, M. Leroux, B. Damilano, S. Rennesson, F. Semond, B. Gayral, C. Brimont, and T. Guillet, Appl. Phys. Lett. 109, 231101 (2016).

${ }^{4}$ T. R. Kuykendall, A. M. Schwartzberg, and S. Aloni, Adv. Mater. 27, 5805 (2015).

${ }^{5}$ N. Alfaraj, R. Aljefri, M. Baier, D. Priante, B. Janjua, A. Prabaswara, T. K. Ng, B. S. Ooi, F. Laquai, and X. Li, in International Workshop on Nitride Semiconductors, Orlando, FL (2016).

${ }^{6}$ J. P. Rojas, G. A. T. Sevilla, N. Alfaraj, M. T. Ghoneim, A. T. Kutbee, A. Sridharan, and M. M. Hussain, ACS Nano 9, 5255 (2015).

${ }^{7}$ N. Alfaraj, A. M. Hussain, G. A. Torres Sevilla, M. T. Ghoneim, J. P. Rojas, A. B. Aljedaani, and M. M. Hussain, Appl. Phys. Lett. 107, 174101 (2015).

${ }^{8}$ M. T. Ghoneim, N. Alfaraj, G. A. Torres-Sevilla, H. M. Fahad, and M. M. Hussain, IEEE Trans. Electron Devices 63, 2657 (2016).

${ }^{9}$ M. T. Ghoneim, N. Alfaraj, G. A. T. Sevilla, and M. M. Hussain, in IEEE 15th International Conference on Nanotechnology (IEEE-NANO), Rome, Italy (2015), pp. 1422-1425.

${ }^{10}$ M. T. Ghoneim, N. Alfaraj, G. A. T. Sevilla, H. M. Fahad, and M. M. Hussain, in 73rd Annual Device Research Conference (DRC), Columbus, OH (2015), pp. 95-96.

${ }^{11}$ M. Gadalla, M. Abdel-Rahman, and A. Shamim, Sci. Rep. 4, 4270 (2014).

${ }^{12}$ A. Hazari, M. Zunaid Baten, L. Yan, J. M. Millunchick, and P. Bhattacharya, Appl. Phys. Lett. 109, 191102 (2016).

${ }^{13}$ C. Zhao, T. K. Ng, R. T. ElAfandy, A. Prabaswara, G. B. Consiglio, I. A. Ajia, I. S. Roqan, B. Janjua, C. Shen, J. Eid, A. Y. Alyamani, M. M. ElDesouki, and B. S. Ooi, Nano Lett. 16, 4616 (2016).

${ }^{14} \mathrm{~F}$. Glas, Wide Band Gap Semiconductor Nanowires 1 (John Wiley and Sons, Inc., 2014) pp. 25-57.

${ }^{15}$ D. Banerjee, S. Sankaranarayanan, D. Khachariya, M. B. Nadar, S. Ganguly, and D. Saha, Appl. Phys. Lett. 109, 031111 (2016).

${ }^{16}$ J. Lähnemann, M. Den Hertog, P. Hille, M. de la Mata, T. Fournier, J. Schörmann, J. Arbiol, M. Eickhoff, and E. Monroy, Nano Lett. 16, 3260 (2016).

${ }^{17}$ P. Wang, Y. Yuan, C. Zhao, X. Wang, X. Zheng, X. Rong, T. Wang, B. Sheng, Q. Wang, Y. Zhang, L. Bian, X. Yang, F. Xu, Z. Qin, X. Li, X. Zhang, and B. Shen, Nano Lett. 16, 1328 (2016).

${ }^{18}$ W. Guo, A. Banerjee, P. Bhattacharya, and B. S. Ooi, Appl. Phys. Lett. 98, 193102 (2011).

${ }^{19}$ H. Okumura, D. Martin, and N. Grandjean, Appl. Phys. Lett. 109, 252101 (2016).

${ }^{20}$ H. Durmaz, D. Nothern, G. Brummer, T. D. Moustakas, and R. Paiella, Appl. Phys. Lett. 108, 201102 (2016).

${ }^{21}$ C. E. Dreyer, A. Alkauskas, J. L. Lyons, J. S. Speck, and C. G. Van de Walle, Appl. Phys. Lett. 108, 141101 (2016).

${ }^{22}$ K. W. Williams, N. R. Monahan, D. D. Koleske, M. H. Crawford, and X.-Y. Zhu, Appl. Phys. Lett. 108, 141105 (2016).

${ }^{23}$ L. Liu, L. Wang, N. Liu, W. Yang, D. Li, W. Chen, Z. C. Feng, Y.-C. Lee, I. Ferguson, and X. Hu, J. Appl. Phys. 112, 083101 (2012).

${ }^{24}$ P. Berdahl, J. Appl. Phys. 58, 1369 (1985).

${ }^{25}$ A. David, C. A. Hurni, N. G. Young, and M. D. Craven, Appl. Phys. Lett. 109, 083501 (2016).

${ }^{26}$ M. G. Kibria, R. Qiao, W. Yang, I. Boukahil, X. Kong, F. A. Chowdhury, M. L. Trudeau, W. Ji, H. Guo, F. Himpsel et al., Adv. Mater. 28, 8388 (2016).

${ }^{27}$ Y. Yong, H. Jiang, X. Li, S. Lv, and J. Cao, Phys. Chem. Chem. Phys. 18, 21431 (2016)
${ }^{28}$ J.-B. Wang, S. R. Johnson, D. Ding, S.-Q. Yu, and Y.-H. Zhang, J. Appl. Phys. 100, 043502 (2006).

${ }^{29}$ C. Zhao, T. K. Ng, N. Wei, A. Prabaswara, M. S. Alias, B. Janjua, C. Shen, and B. S. Ooi, Nano Lett. 16, 1056 (2016).

${ }^{30}$ G. Martin, A. Botchkarev, A. Rockett, and H. Morkoc, Appl. Phys. Lett. 68, 2541 (1996).

${ }^{31}$ J. Piprek, Phys. Status Solidi A 207, 2217 (2010).

${ }^{32}$ S. Jain, M. Willander, J. Narayan, and R. Van Overstraeten, J. Appl. Phys. 87, 965 (2000).

${ }^{33}$ A. Gorgis, T. Flissikowski, O. Brandt, C. Chèze, L. Geelhaar, H. Riechert, and H. T. Grahn, Phys. Rev. B 86, 041302 (2012).

${ }^{34}$ Q. Dai, M. F. Schubert, M. H. Kim, J. K. Kim, E. F. Schubert, D. D. Koleske, M. H. Crawford, S. R. Lee, A. J. Fischer, G. Thaler, and M. A. Banas, Appl. Phys. Lett. 94, 111109 (2009).

${ }^{35} \mathrm{~S}$. Adachi, Optical Constants of Crystalline and Amorphous Semiconductors: Numerical Data and Graphical Information (Kluwer Academic Publishing, 1999), p. 183.

${ }^{36}$ Y. C. Shen, G. O. Mueller, S. Watanabe, N. F. Gardner, A. Munkholm, and M. R. Krames, Appl. Phys. Lett. 91, 141101 (2007).

${ }^{37}$ N. Shimosako, Y. Inose, H. Satoh, K. Kinjo, T. Nakaoka, T. Oto, K. Kishino, and K. Ema, J. Appl. Phys. 118, 175702 (2015).

${ }^{38}$ M. Fox, Optical Properties of Solids (Oxford University Press, 2010).

${ }^{39}$ M. J. Davies, T. J. Badcock, P. Dawson, M. J. Kappers, R. A. Oliver, and C. J. Humphreys, Appl. Phys. Lett. 102, 022106 (2013).

${ }^{40}$ C. Martinez, N. Stanton, A. Kent, D. Graham, P. Dawson, M. Kappers, and C. Humphreys, J. Appl. Phys. 98, 053509 (2005).

${ }^{41}$ S. Watanabe, N. Yamada, M. Nagashima, Y. Ueki, C. Sasaki, Y. Yamada, T. Taguchi, K. Tadatomo, H. Okagawa, and H. Kudo, Appl. Phys. Lett. 83, 4906 (2003).

${ }^{42}$ P. Wen, S. Zhang, J. Liu, D. Li, L. Zhang, Q. Sun, A. Tian, K. Zhou, T. Zhou, and H. Yang, J. Appl. Phys. 119, 213107 (2016).

${ }^{43}$ P. G. Eliseev, P. Perlin, J. Lee, and M. Osiński, Appl. Phys. Lett. 71, 569 (1997).

${ }^{44}$ M. Weinstein, J. Opt. Soc. Am. 50, 597 (1960).

${ }^{45}$ P. Landsberg and G. Tonge, J. Appl. Phys. 51, R1 (1980).

${ }^{46}$ Y.-H. Cho, G. H. Gainer, A. J. Fischer, J. J. Song, S. Keller, U. K. Mishra, and S. P. DenBaars, Appl. Phys. Lett. 73, 1370 (1998).

${ }^{47}$ M. Benaissa, W. Sigle, T. K. Ng, R. El Bouayadi, P. A. van Aken, S. Jahangir, P. Bhattacharya, and B. S. Ooi, Appl. Phys. Lett. 106, 101910 (2015).

${ }^{48}$ A. Mohanta, S.-F. Wang, T.-F. Young, P.-H. Yeh, D.-C. Ling, M.-E. Lee, and D.-J. Jang, J. Appl. Phys. 117, 144503 (2015).

${ }^{49}$ F. C.-P. Massabuau, P. Chen, M. K. Horton, S. L. Rhode, C. X. Ren, T. J. O'Hanlon, A. Kovcs, M. J. Kappers, C. J. Humphreys, R. E. DuninBorkowski, and R. A. Oliver, J. Appl. Phys. 121, 013104 (2017).

${ }^{50}$ M. K. Horton, S. Rhode, S.-L. Sahonta, M. J. Kappers, S. J. Haigh, T. J. Pennycook, C. J. Humphreys, R. O. Dusane, and M. A. Moram, Nano Lett. 15, 923 (2015).

${ }^{51}$ S. Jahangir, M. Mandl, M. Strassburg, and P. Bhattacharya, Appl. Phys. Lett. 102, 071101 (2013).

${ }^{52}$ R. Ivanov, S. Marcinkeviius, Y. Zhao, D. L. Becerra, S. Nakamura, S. P. DenBaars, and J. S. Speck, Appl. Phys. Lett. 107, 211109 (2015).

${ }^{53}$ T. Li, A. M. Fischer, Q. Y. Wei, F. A. Ponce, T. Detchprohm, and C. Wetzel, Appl. Phys. Lett. 96, 031906 (2010).

${ }^{54}$ T. Badcock, P. Dawson, M. Davies, M. Kappers, F.-P. Massabuau, F. Oehler, R. Oliver, and C. Humphreys, J. Appl. Phys. 115, 113505 (2014).

${ }^{55}$ C. Zhao, T. K. Ng, A. Prabaswara, M. Conroy, S. Jahangir, T. Frost, J. O'Connell, J. D. Holmes, P. J. Parbrook, P. Bhattacharya, and B. S. Ooi, Nanoscale 7, 16658 (2015).

${ }^{56}$ A. Armstrong, Q. Li, Y. Lin, A. Talin, and G. Wang, Appl. Phys. Lett. 96, 163106 (2010). 